\title{
Analysis of the Results of Use of Bone Graft and Reconstruction Cages in a Group of Patients with Severe Acetabular Bone Defects
}

\author{
Ainhoa Toro-Ibarguen, Ismael Auñón-Martín, Emilio Delgado-Díaz, \\ Jose Alberto Moreno-Beamud, Miguel Ángel Martínez-Leocadio, \\ Andrés Díaz-Martín, and Luciano Candel-García
}

Orthopaedic and Traumatology Surgery, Hospital 12 de Octubre, 28041 Madrid, Spain

Correspondence should be addressed to Ainhoa Toro-Ibarguen; aonia.orot@gmail.com

Received 29 July 2014; Accepted 17 September 2014; Published 30 September 2014

Academic Editor: Palaniappan Lakshmanan

\begin{abstract}
Copyright (C) 2014 Ainhoa Toro-Ibarguen et al. This is an open access article distributed under the Creative Commons Attribution License, which permits unrestricted use, distribution, and reproduction in any medium, provided the original work is properly cited.

Introduction. Rings and cages are indicated for use in revision total hip with severe bone loss. Material and Methods. A retrospective study was performed on 37 acetabular revision cases with an average age at revision of 67.8 years. According to Paprosky classification, 54\% grade II and 46\% grade III. We used two types of cages, Protrusio and Contour cage. We used 23 standard liners and 14 dual mobility cups. Results. The average follow-up was 5.4 years. The mean Merlé-d'Aubigné score improved from 5.48 to 10.5 points $(P<0.05)$. There were 10 nerve palsies, 6 rings that lost fixation, 10 dislocations, and 4 infections. The need for reoperation for any reason rose to $32 \%(12 / 37)$. Success, defined as a stable reconstruction, was $73 \%$. We found that, using a dual mobility cup cemented into the cage, the dislocation rate and revision rate came down $(P<0.05)$. Conclusions. The treatment of severe acetabular defects using bone graft and reconstruction cages is a viable option. The use of a dual mobility cup cemented into the cage could avoid dislocations and the insertion of the ischial flap inside the ischial portion of the acetabulum for further ring stability and protection of the sciatic nerve.
\end{abstract}

\section{Introduction}

The demand for primary total hip arthroplasty (THA) is expected to increase over the next several decades, due to the aging population and the obesity crisis [1]. The need for THA is expected to grow $174 \%$ to 572,000 primary THAs per year by 2030 in the United States [1]. Consequently, the demand for revision total hip arthroplasty will also increase exponentially. It is estimated that total hip arthroplasty revisions will double every 10 years [2].

The most common indications for acetabular revision include hip instability, aseptic loosening, periprosthetic osteolysis, and infection $[2,3]$, with the acetabular component involved in $>50 \%$ of revisions [3]. Acetabular bone loss can be found in any of the revisions of THA and is one of the factors to be taken into account when determining treatment. There are multiple options for the treatment of these defects, each of which have their strengths and weaknesses, as all have common goals such as bone stock rebuild and provide mechanical stability with a maximum host bone contact [4]. Rings and cages are indicated for use in revision THA with severe bone loss, as those described in Paprosky classification, as types II and III [5]. The antiprotrusio cages are designed to manage extended pelvic defects by bridging large bone gaps and protecting the grafts filled to increase the bone stock [6].

We analyze the perioperative and intermediate-term complications and outcome of a consecutive series of 37 reconstruction rings and extensive bone-grafting techniques.

\section{Material and Methods}

A retrospective single-center study was performed between January 2003 and December 2011. This study included 37 
patients who underwent acetabular revision using reconstruction rings, during the inclusion period. There were 25 females and 12 males, with an average age at revision of 67.8 years (range, 29-90 years). 17 were left hips and 20 were right.

In 24 cases (65\%), primary hip arthroplasty was indicated because of hip osteoarthritis (Figure 1).

The average time from index arthroplasty to the acetabular revision was 13.4 years (range, 1-27 years). The time from the last procedure to acetabular revision was 7.4 years (range, $0.2-27$ years). The number of previous surgeries on the hip in question averaged 1.9 and was 1 (16), 2 (12), 3 (5), 4 (3), and 5 (1). We revised 25 hips for aseptic loosening, one for infection, 3 for acetabular fracture, and eight for recurrent dislocation associated with acetabular loosening.

This procedure was indicated preoperatively based on an evaluation of clinical and radiological records, with potential intraoperative adjustment. A posterolateral approach was used in 32 cases $(87 \%)$, in one case with femoral trochanteric osteotomy, to obtain better visualization of a Paprosky type IIIb. In 5 cases (13\%) the surgeons preferred a Hardinge transgluteal approach. The hip joint was exposed and the acetabular component was removed and the femoral component was tested. Once the acetabulum had been cleaned, the severity of the acetabular defect was graded using the Paprosky classification system [5] (Figure 2).

In four cases angiography was needed to check the anatomical relation between the implant or cement and the pelvic vessels to ascertain their proximity, being the collaboration of vascular surgeons necessary during the intervention. Bone defects were filled with bone graft, using in all cases morselized bones that were small chunks of $0.5 \mathrm{~cm}$, instead of slurry of bone which would make impaction impossible. Morselized allograft bone was firmly impacted with smooth acetabular impaction domes. In two cases structural allografts were used, which were composed of one or more femoral heads. Intraoperatively, the graft was reamed and sized to closely press-fit the residual host bone. Of the 37 cages used in this study, 15 were performed with a Protrusio cage [DePuy Orthopaedics, Inc, Warsaw, IN] and 22 with Contour types [Smith and Nephew Richards, Memphis, TN]. A cage was shaped to provide optimal congruity to the grafted acetabulum. The superior and inferior flanges were bended in order to comply with the individual anatomy of the acetabular region and to maximize the stability of the cage. Iliac fixation was obtained using 2 to 5 cancellous screws, which were placed first in the acetabular dome. The ischiatic flange was fixed using 1 to 3 cancellous screws in 27 reconstruction cages. In 1 cage the inferior flange was slotted into the ischium and in nine cases it was bent to engage the tear drop medially. We used on average 6.6 screws (range, 3-9 screws) to secure the reconstruction cages. After secure fixation of the reconstruction cage, we cemented an all polyethylene acetabular component into the cage in 23 hips. In 14 hips the device used was a dual mobility cup, Polar Cup [Smith and Nephew Richards, Memphis, TN]. The femoral component also was revised in 9 of the 37 cases.

The Postel Merlé D’Aubigné (PMA) score [7] was used to assess patient function. We also noted any medical and surgical complications. According to Goodman et al. [8], we

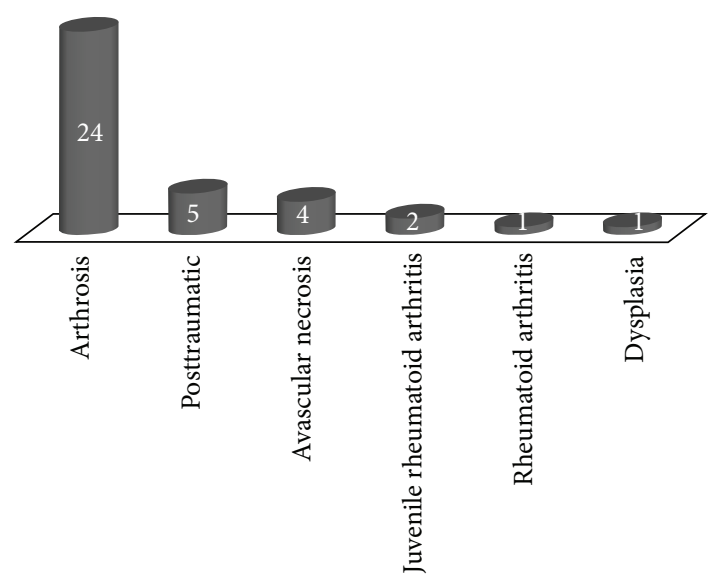

FIGURE 1: Indications that led to primary hip arthroplasty.

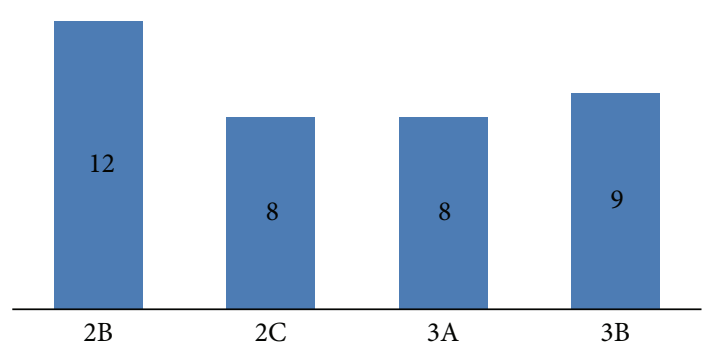

FIgURE 2: Distribution of the patients depending on the acetabular bone loss using the Paprosky classification.

defined success of the reconstruction as a stable, nondislocating, nonmigrating hip with incorporation of the acetabular bone graft without fracture or resorption that has had no further surgery on the acetabular side. Because several cases required revision of the acetabular cup only, without removal of the reconstruction ring, we also determined a second measure of success in which the acetabular cup was revised, leaving the ring and incorporated bone graft intact.

The radiological assessment was performed on an $\mathrm{A} / \mathrm{P}$ view of the pelvis and A/P and lateral views of the hip. Failure of an acetabular cage was defined as a change of more than $5^{\circ}$ in the inclination of the reinforcement ring, migration of more than $5 \mathrm{~mm}$ in either the vertical or horizontal direction, or breakage of the material [9]. The reference point for measuring vertical migration was the inferior aspect of the teardrop, and the Kohler line was the reference for measuring horizontal migration [9]. The bone/graft interface was evaluated using the three-zone classification proposed by DeLee and Charnley [10] to locate radiolucent lines. Radiolucent lines were defined as either simple (less than $1 \mathrm{~mm}$ thick, stable, less than $50 \%$ of area) or complete (less than $1 \mathrm{~mm}$ thick, stable, covering the entire surface area) or complex ( $2 \mathrm{~mm}$ or more in thickness, with or without progression, independent of thickness, location, and extent). The outcome of grafting was evaluated seeing incorporation, resorption, or fracture of the bone graft. Graft incorporation was indicated by continuation of trabecular lines from the graft into host bone without resorption or fracture $[11,12]$. 


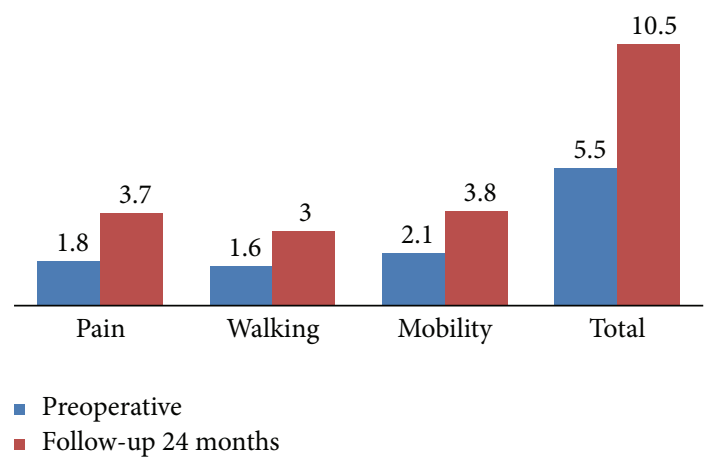

Figure 3: Change in the Postel Merlé d'Aubigné from preoperative to follow-up. The PMA pain score went from 1.8 to 3.7, the walking score went from 1.6 to 3.0 and the mobility score went from 2.1 to 3.8 , on average.

A critical $P$ value less than 0.05 was used for all statistical comparisons. All analyses were performed using STATA/SE v10.

\section{Results}

Follow-up averaged 5.4 years; the longest follow-up was 12.5 years. Only one patient died of causes unrelated to the process at a mean follow-up of 24 months.

Preoperative PMA functional scores [7] averaged $5.48 \pm$ 2.41. We observed an improvement $(P<0.01)$ in the postoperative score with an average PMA of $10.55 \pm 3.82$ (Figure 3 ). Every component of the PMA changed significantly $(P<$ 0.001). There was no significant effect of gender, number of previous surgeries, use of a dual mobility cup, or severity of preoperative bone loss on the functional results. Patients less than 65 years old had better functional scores than patients above 65 years of age $(P<0.005)$.

Thirty-four (92\%) structural allografts and morselized cancellous grafts healed uneventfully without fracture or resorption, so that, radiographically, there was a continuous trabecular pattern with host bone. Only 3 patients (8\%), those cases with septic acetabular loosening, had progressive radiolucent lines (8\%) [10].

We reviewed the 37 patients for any perioperative or intermediate complications. Fourteen of the 37 cases (38\%) had no perioperative complications.

There was a 77-year-old woman who was revised at 44 months because of a septic loosening that was associated to recurrent dislocations. The radiograph showed that one of the superior flanges had fractured through the screw holes. A two-stage exchange was deemed necessary with implant of a total femur replacement and a dual mobility cup cemented into a reconstruction cage. At 2 months postoperatively two early dislocations occurred; therefore a new revision to a constrained liner was necessary, with no further complications.

Six rings lost fixation (16\%), 3 septic and 3 aseptic loosening: 3 of them were revised and 1 is awaiting revision. (i) Two revised hips were associated with infection. One of them was the patient explained above. A two-stage exchange was also necessary for the other patient, with implant of a dual mobility cup cemented into a new Contour cage and a total femur replacement. The other septic loosening patient refused to have surgery; he is actually with antibiotherapy.

(ii) There were three aseptic loosenings, of which only one was revised at 84 months. In this case a Protrusio cage lost fixation and impinged the sciatic nerve, so a revision to another cage and neurolysis of the sciatic nerve were necessary. One is awaiting revision. The last one had an early dislocation that was reduced recurrence-free and an aseptic loosening at 36 months postoperatively, but he refused to have surgery.

Two rings possibly were lost with loss of ischial screw fixation in one of them. The other one was a cage with rupture and migration of an ischial screw which impinged the sciatic nerve 2 months postoperatively, requiring the prompt removal of the screw, leaving the cage intact. Both of them had good results in the overall PMA at 104- and 24-month follow-up, respectively. One acetabular cup has become loosened 24 months later and has been revised, leaving the ring intact, with no further complications.

We found no association between the number of previous surgeries and both radiographic failure and loosening undergoing revision. The extent of preoperative bone loss as determined by the Paprosky classifications (type III) [5] was associated with an increased rate of loosening or failure undergoing revision $(P<0.05)$. The acetabular component abduction angles were similar for loosened and unloosened cages $\left(48,7^{\circ}+/-5,4^{\circ}\right.$ and $48,5^{\circ}+/-5,7^{\circ}$, resp. $)$. We found no association between age at revision, number of screws used, size of the reconstruction cage, and use of a dual mobility cup with both radiographic failure and loosening undergoing revision.

There were 4 deep infections (10.8\%), three septic acetabular loosenings, explained above, and one further deep infection, successfully treated with irrigation, debridement, and retention of the implants, with no further complications.

Ten hips had dislocations, only one having a dual mobility cup. There were 7 cases of early dislocation (before 3 months postoperatively): 3 of them were reduced without additional surgery and remained recurrence-free. And there were 3 cases of late dislocations ( $>3$ months). The overall postoperative dislocation rate at the end of FU was $27 \%$ of which 7 (18.9\%) needed further revision. One case was associated with septic loosening as explained above. Another two cases were revised to dual mobility cups, without further complications. In two patients aged 89 and 90, respectively, a resection arthroplasty was left. One of them died of causes unrelated to the process 3 months after the surgery. Another one was revised to a standard cemented liner and dislocated two times again; therefore, another revision was needed to a dual mobility cup, without recurrence. The last patient was a dislocation of a dual mobility cup cemented into a cage 3 months postoperatively. After several attempts to reduction, we found the prosthesis disassembled, so a revision to a 
constrained liner was necessary in this case. We found an inverse association between the use of dual mobility cups and both dislocation rate $(P<0.05)$ and dislocation undergoing revision $(P<0.05)$.

There were 9 sciatic nerve palsies and 1 femoral nerve palsy. Three cases of sciatic impingement were observed and needed revision: two cases explained above and another case were associated with fracture of the ischium 12 months later with posterior fibrosis of the sciatic nerve that needs neurolysis. The remaining 6 cases, except one, were associated with application of the reconstruction ring on the lateral surface of the ischium and subsequent screw fixation. Full recovery occurred in all cases, except two cases with partial recovery and 1 case with no recovery of the sciatic palsy.

One case had a femoral deep venous thrombosis that provoked an acute arterial ischemia in the popliteal artery. A thrombectomy had to be made.

We observed two material ruptures (screw or cage): the above-mentioned Contour superior flange fracture with septic loosening and the other above mentioned ischial screw fracture that impinged the sciatic nerve needing further revision. The latter one did not cause migration of the cage; therefore it was not necessary to remove it.

In summary, two constrained liners were used because of recurrent dislocations.

The need for reoperation for any reason was $32 \%$, twelve hips (Figure 4).

Given the above criteria for success dictated by Goodman et al. [8] 19 of the 37 hips (51.4\%) were successful, and 18 of the 37 hips (48.6\%) were deemed to be unsuccessful. However, 8 of these 18 "unsuccessful" rings were left in situ: two patients who refused revision to a new reconstruction cage for loosening cages, 2 patients with one episode of early dislocation that were reduced recurrence-free, 2 revisions for sciatic impingement, where the cages were left intact, one deep infection which was successfully treated with antibiotherapy, and one acetabular cup loosening. In all these "failed" cases, the bone graft and reconstruction ring have been left in situ and thus may be considered successful in the fact that they restored bone stock for future revision, if necessary [8]. Therefore, we could consider that the success rate of this reconstruction is 27 of 37 hips (73\%). There were no differences in the outcome or rate of complications when comparing the Protrusio rings and the Contour rings.

\section{Discussion}

The presence of severe bone loss is an indication for an acetabular reconstruction with the use of a metal reinforcement ring and bone graft [13-15]. A reinforcement cage is designed to manage extended pelvic defects by bridging large bone gaps and protecting the grafts filled to increase the bone stock in case of future revision [6]. The major advantage of rings and cages is the ability to cement a liner in any position independent of the cage position and the elution of local antibiotics from the cement [2]. The lack of bony ingrowth and biological fixation exposed them to cyclical loading resulting in failure of hardware, typically screw breakage or migration of the ischial flange $[8,13,16]$, with this being their major

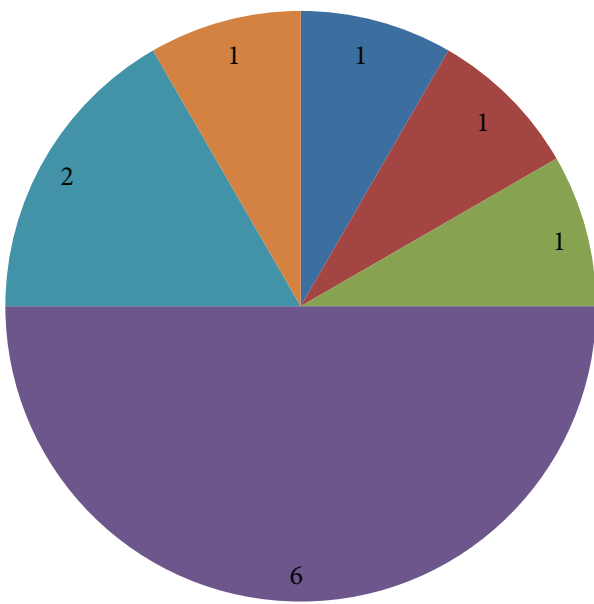

- Septic loosening

- Septic loosening + dislocation

- Aseptic loosening + sciatic nerve impingement

FIGURE 4: Need for reoperation for any reason.

disadvantage. Despite this, midterm results with the use of a cage have been satisfactory, with a survival rate that ranges between $75 \%$ with 15 -year follow-up [8] and $92,4 \%$ for 68 antiprotrusio cages after an average follow-up time of 13 years [17].

We noted a modest improvement in the clinical outcome using the PMA score [7]. Our data regarding pain relief and functional improvement support the observation made by others that improvement in function was limited $[8,12]$. Goodman et al. [8] reported a modest functional improvement in their series probably not reflective of reconstruction success given the limitations present preoperatively because of multiple previous surgeries and widespread arthritis of the joints of the lower extremities or debilitating systemic disease or bilateral reconstructions. Therefore, they defined [8] success of reconstruction as a stable, nondislocating, nonmigrating hip that has had no further surgery on the acetabular side. Our data, with a success rate of $73 \%$ (27 of 37 hips), are similar to Goodman et al's [8], which reported a success rate of $76 \%$.

The results and complications of our study are comparable with other reports describing the use and outcome of reconstruction rings. The perioperative and intermediate rate of complications in our study was $62 \%$, similar to Goodman et al. [8] with 56\% (34/61) and higher than Philippe et al. [18] with $38 \%$. The high complication rate associated with these cases reflects the very large acetabular bone deficiencies that must be replaced; the extensive scarring from previous surgery; and the elevated age at revision and high prevalence of local or systemic disorders [8].

Reconstruction with an antiprotrusio cage has a reported failure rate including radiographic loosening that ranges between 0 and $24 \%$ [17-25]. The reported rates of revision ranged from 0 to $16 \%[8,17-25]$. The incidence of failure or radiographic loosening in our study was $16 \%$ at an average 
of 5.4 years postoperatively, with the need for revision for this cause being $8.1 \%$ (2.7\% without infection). These rates are comparable to other studies like Bostrom et al. [20] with a revision rate of $6 \%$ (3\% without infection) and an incidence of radiographic loosening of $16 \%$ or Goodman et al. [8] which revealed a revision rate of $6.6 \%$ and a radiographic loosening or failure of $11.5 \%$. Sembrano and Cheng [26] reported that no single preoperative factor (age, gender) or intraoperative factor (number of screws used, size of the reconstruction cage, and number of previous surgeries or restoration of an optimal center of rotation) predicted cage failure. In fact, in our analysis of different factors, we were not able to identify a single preoperative or intraoperative factor that correlated with failure. We found only one association between preoperative classification of bone loss and radiographic failure and loosening undergoing revision $(P=0.03)$. As Perka and Ludwig [12] reported, we found an increased rate of revision because of cage failure in Paprosky type III defects.

The all-cause rate of acetabular revision described in the literature concerning antiprotrusio cage reconstructions varies from $5 \%$ to $24 \%[20-22,24]$. The need for reoperation for any reason was $32 \%$ (twelve hips) in our study, three for failure (one aseptic and two infected, one of them associated with dislocation), six for recurrent dislocation, two for sciatic impingement, and one for cup loosening. Our extremely high rates of revision are probably due to the high number of dislocations $(7 / 37)$ or sciatic nerve palsies $(3 / 37)$ that needed revision. It is well known that patients undergoing this form of surgery are at the risk of instability because of many causes, like repeated hip operations, function of abductors [16], position of the components, or cooperation of patient and others. The rate of implant instability during revision of total hip replacement is highly variable in the literature, with recent publications showing a dislocation rate varying from 7.4 to $17.2 \%[17,18,20,21,27]$. We reported a $39 \%$ dislocation rate (9/23) during the analysis of 23 reconstruction cages using a standard cemented liner, a rate that is unacceptably high and should not be accepted in modern orthopaedic practice. There are many probable causes that may have caused this huge dislocation rate in our series. We observed that, by using a dual mobility cup cemented into the cage, our rates improved from $39 \%$ to $7.1 \%(1 / 14)$. Schneider et al. [19] observed a $10 \%(10 / 96)$ dislocation rate at a mean follow-up of 41 months in 96 revisions using a reconstruction device associated with a dual mobility cup cemented into the 13 cages, very similar to our series. We observed that the dislocation rate and dislocation undergoing revision decreased using a cemented dual mobility cup into the cage $(P=0.03)$.

We observed a high rate of sciatic nerve palsies $(24 \%)$ undergoing revision (8.1\%); one of them associated with an aseptic loosening of the cage that impinged the sciatic nerve. This could be explained by the following: the acetabular component to be removed often is associated with marked protrusio, fractured and migrated internal fixation, and cement and fibrous adhesions after the previous surgeries can be found. These factors often demand extensive exposure and retraction, which can be associated with stretching of the branches of the sciatic nerve $[4,8,25]$. Fixing the flange to the lateral surface of the ischium with screws, as we did, demands more exposure and retraction near the sciatic nerve; furthermore, the flange could act as an irritant, subsequently leading to sciatica [8]. Slotting the inferior flange into the ischium is a technically demanding procedure but provides several advantages: a more stable fixation of the cage can be obtained in a more horizontal position. Moreover, the risk of sciatic nerve injury can be significantly decreased $[4,8,25]$, because the ischiatic flap is inserted inside the ischium.

Finally, we only observed two material ruptures (5.4\%). The major concern with standard acetabular cages is the lack of a porous coating for bone ingrowth. Consequently, the potential for biologic, long-term fixation through osseointegration is not predictable, and a high incidence of hardware failure due to screw breakage or ischial flange migration has been reported at midterm follow-up [6]. Our series had a low rate of material rupture, which could explain the high rate of graft uptake in our series. The cages spanning the bone defect allow primary stability and protect the graft from resorption due to mechanical overload, enabling osseous integration [4].

Our study had significant limitations. First, it was a retrospective study without a control group. Second, two reconstruction devices were used in the patients and may have confused the results. Third, midterm follow-up limited our ability to generate definitive conclusions with a power analysis, especially in relation to cup loosening. Fourth, there were only 37 , so larger studies with longer follow-up periods are needed.

Although a longer follow-up is required before reaching definitive conclusions, our preliminary results indicate that the treatment of severe acetabular defects by this technique is a viable option and we emphasized the need to use a dual mobility cup cemented into the cage to improve the device stability. Inserting the ischial flange into the ischium could be a possibility to decrease nerve palsy injury and to improve mechanical stability [25].

\section{Conflict of Interests}

The authors declare that they have no conflict of interests concerning this paper.

\section{Acknowledgment}

Thanks are due to Mr. Martin J. Smyth, B.A., for his help in revising the English language.

\section{References}

[1] S. J. Nho, S. M. Kymes, J. J. Callaghan, and D. T. Felson, “The burden of hip osteoarthritis in the United States: epidemiologic and economic considerations," The Journal of the American Academy of Orthopaedic Surgeons, vol. 21, supplement 1, pp. S1S6, 2013.

[2] N. P. Sheth, C. L. Nelson, B. D. Springer, T. K. Fehring, and W. G. Paprosky, "Acetabular bone loss in revision total hip arthroplasty: evaluation and management," Journal of the 
American Academy of Orthopaedic Surgeons, vol. 21, no. 3, pp. 128-139, 2013.

[3] G. K. Deirmengian, B. Zmistowski, J. T. O’Neil, and W. J. Hozack, "Management of acetabular bone loss in revision total hip arthroplasty," Journal of Bone and Joint Surgery A, vol. 93, no. 19, pp. 1842-1852, 2011.

[4] D. Regis, A. Sandri, I. Bonetti, O. Bortolami, and P. Bartolozzi, "Acminimum of 10-year follow-up of the Burch-Schneider cage andcbulk allografts for the revision of pelvic discontinuity," The Journal of Arthroplasty, vol. 27, no. 6, pp. 1057.e1-1063.e1, 2012.

[5] W. G. Paprosky, P. G. Perona, and J. M. Lawrence, "Acetabular defect classification and surgical reconstruction in revision arthroplasty: a 6-year follow-up evaluation," Journal of Arthroplasty, vol. 9, no. 1, pp. 33-44, 1994.

[6] D. Regis, A. Sandri, and I. Bonetti, "Acetabular reconstruction with the Burch-Schneider antiprotrusio cage and bulk allografts: minimum 10-year follow-up results," BioMed Research International, vol. 2014, Article ID 194076, 9 pages, 2014.

[7] R. Merle D’Aubigné, "Numerical classification of the function of the hip," Revue de Chirurgie Orthopédique et Réparatrice de l'appareil Moteur, vol. 56, pp. 481-486, 1970.

[8] S. Goodman, H. Saastamoinen, N. Shasha, and A. Gross, "Complications of ilioischial reconstruction rings in revision total hip arthroplasty," Journal of Arthroplasty, vol. 19, no. 4, pp. 436446, 2004.

[9] F. Bonnomet, P. Clavert, P. Gicquel, Y. Lefèbvre, and J.-F. Kempf, "Reconstruction by graft and metallic reinforcement device in severe aseptic acetabular loosening: 10 years survivorship analysis," Revue de Chirurgie Orthopedique et Reparatrice de l'Appareil Moteur, vol. 87, no. 2, pp. 135-146, 2001.

[10] J. G. DeLee and J. Charnley, "Radiological demarcation of cemented sockets in total hip replacement," Clinical Orthopaedics and Related Research, vol. 121, pp. 20-32, 1976.

[11] T. Azuma, H. Yasuda, K. Okagaki, and K. Sakai, "Compressed allograft chips for acetabular reconstruction in revision hip arthroplasty," Journal of Bone and Joint Surgery Series B, vol. 76, no. 5, pp. 126-137, 1994.

[12] C. Perka and R. Ludwig, "Reconstruction of segmental defects during revision procedures of the acetabulum with the BurchSchneider anti-protrusio cage," Journal of Arthroplasty, vol. 16, no. 5, pp. 568-574, 2001.

[13] E. Winter, M. Piert, R. Volkmann et al., "Allogeneic cancellous bone graft and a burch-schneider ring for acetabular reconstruction in revision hip arthroplasty," Journal of Bone and Joint Surgery-Series A, vol. 83, no. 6, pp. 862-867, 2001.

[14] T. J. Gill, J. B. Sledge, and M. E. Müller, “The Burch-Schneider anti-protrusio cage in revision total hip arthroplasty: indications, principles and long-term results," Journal of Bone and Joint Surgery, vol. 80, no. 6, pp. 946-953, 1998.

[15] A. Bergmann, E. Heisel, and E. Fritsch, "Erfahrungen mit metallischen Abstützringen in Kombination mit zementierten Polyäthylenpfannen bei Hüftendoprothesenwechseln und mögliche Alternativen," Orthop Praxis, vol. 27, pp. 206-211, 1991.

[16] Y. Kosashvili, D. Backstein, O. Safir, D. Lakstein, and A. E. Gross, "Acetabular revision using an anti-protrusion (ilio-ischial) cage and trabecular metal acetabular component for severe acetabular bone loss associated with pelvic discontinuity," Journal of Bone and Joint Surgery, vol. 91, no. 7, pp. 870-876, 2009.

[17] A. Coscujuela-Mañá, F. Angles, C. Tramunt, and X. Casanova, "Burch-Schneider antiprotrusio cage for acetabular revision: a
5- to 13-year follow-up study," Hip International, vol. 20, no. S7, pp. S112-S118, 2010.

[18] R. Philippe, O. Gosselin, J. Sedaghatian et al., "Acetabular reconstruction using morselized allograft and a reinforcement ring for revision arthroplasty with Paprosky type II and III bone loss: survival analysis of 95 hips after 5 to 13 years," Orthopaedics and Traumatology: Surgery and Research, vol. 98, no. 2, pp. 129137, 2012.

[19] L. Schneider, R. Philippot, B. Boyer, and F. Farizon, "Revision total hip arthroplasty using a reconstruction cage device and a cemented dual mobility cup," Orthopaedics and Traumatology: Surgery and Research, vol. 97, no. 8, pp. 807-813, 2011.

[20] M. P. Bostrom, A. P. Lehman, R. L. Buly, S. Lyman, and B. J. Nestor, "Acetabular revision with the contour antiprotrusio cage: 2- to 5-year followup," Clinical Orthopaedics and Related Research, no. 453, pp. 188-194, 2006.

[21] H. Pieringer, V. Auersperg, and N. Böhler, "Reconstruction of severe acetabular bone-deficiency: the Burch-Schneider antiprotrusio cage in primary and revision total hip arthroplasty," The Journal of Arthroplasty, vol. 21, no. 4, pp. 489-496, 2006.

[22] D. J. Berry and M. E. Muller, "Revision arthroplasty using an anti-protrusio cage for massive acetabular bone deficiency," Journal of Bone and Joint Surgery Series B, vol. 74, no. 5, pp. 711$715,1992$.

[23] M. Kerboull, M. Hamadouche, and L. Kerboull, "The Kerboull acetabular reinforcement device in major acetabular reconstructions," Clinical Orthopaedics and Related Research, no. 378, pp. 155-168, 2000.

[24] K. Kawanabe, H. Akiyama, E. Onishi, and T. Nakamura, "Revision total hip replacement using the Kerboull acetabular reinforcement device with morsellised or bulk graft," Journal of Bone and Joint Surgery -Series B, vol. 89, no. 1, pp. 26-31, 2007.

[25] J. Lamo-Espinosa, J. Duart Clemente, P. Díaz-Rada, J. PonsVillanueva, and J. R. Valentí-Nín, "The Burch-Schneider antiprotrusio cage: medium follow-up results," Musculoskeletal Surgery, vol. 97, no. 1, pp. 31-37, 2013.

[26] J. N. Sembrano and E. Y. Cheng, "Acetabular cage survival and analysis of factors related to failure," Clinical Orthopaedics and Related Research, vol. 466, no. 7, pp. 1657-1665, 2008.

[27] A. L. Whaley, D. J. Berry, and W. Scott Harmsen, "Extra-large uncemented hemispherical acetabular components for revision total hip arthroplasty," Journal of Bone and Joint Surgery-Series A, vol. 83, no. 9, pp. 1352-1357, 2001. 


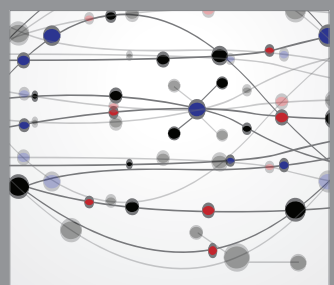

The Scientific World Journal
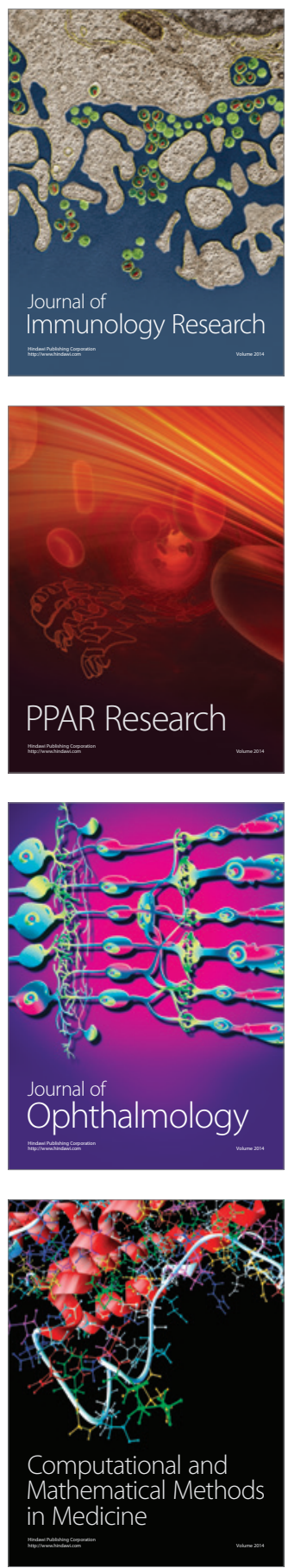

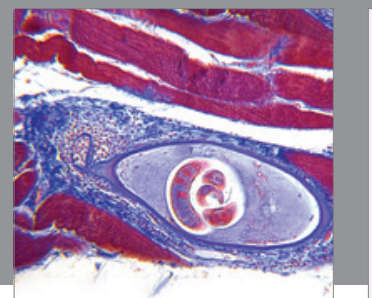

Gastroenterology

Research and Practice
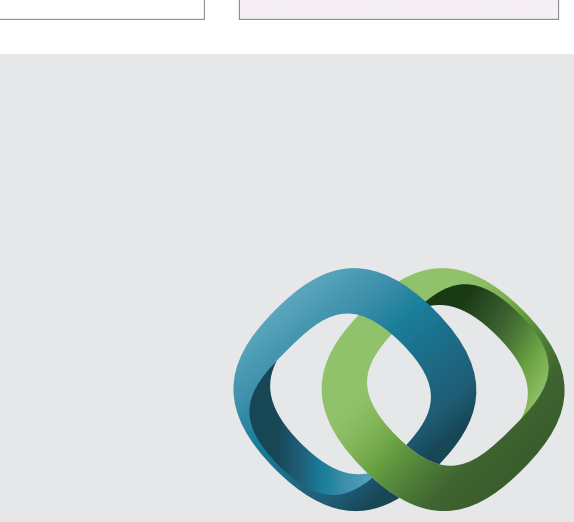

\section{Hindawi}

Submit your manuscripts at

http://www.hindawi.com
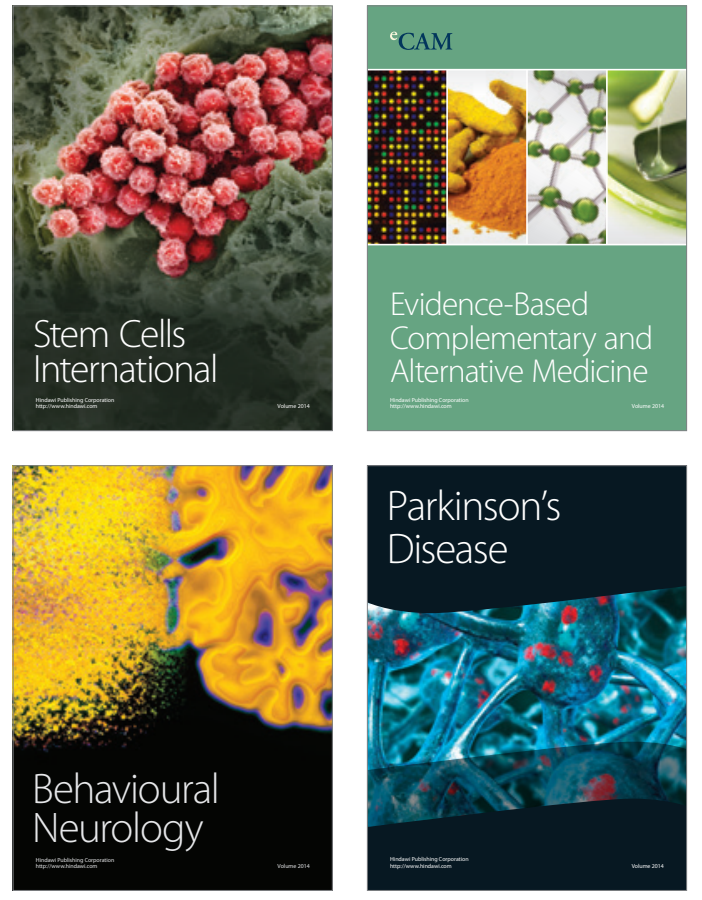
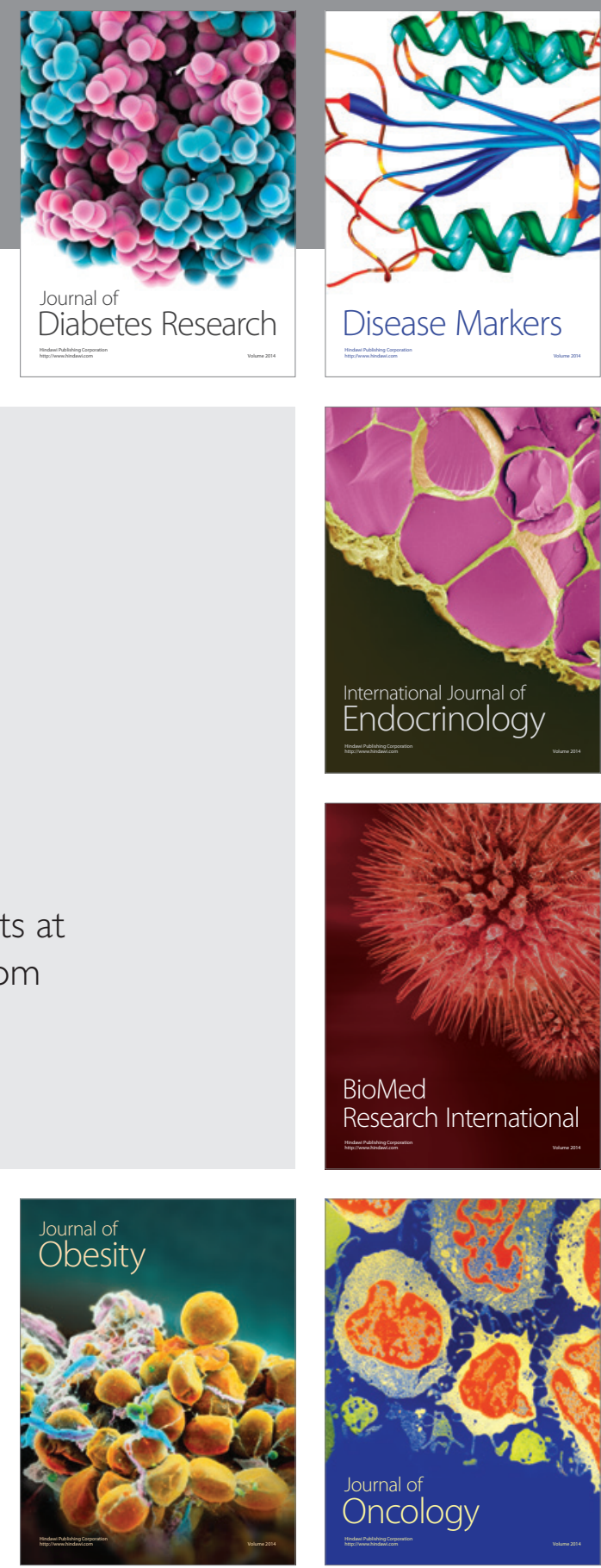

Disease Markers
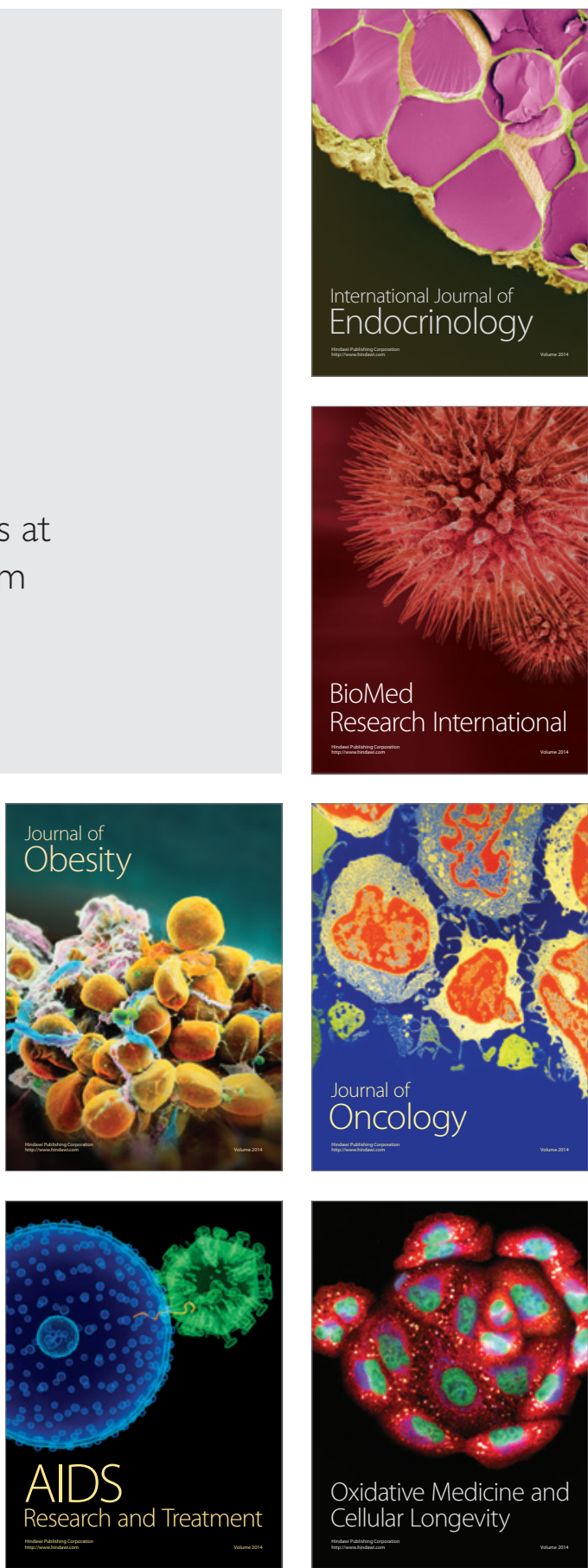